\title{
Knowledge, Attitude, and Practice of Type 2 Diabetes Mellitus Patients on Ramadan Fasting at Hospital Universiti Sains Malaysia
}

\author{
Nor Azwany Yaacob, MCommMed; Abdul Aziz Al-Safi Ismail, PhD; \\ Wan Mohammad Wan Bebakar, FRCP \\ Kelantan, Malaysia
}

\begin{abstract}
Objective: To assess the level of knowledge, attitude, and practice of type 2 diabetes patients regarding Ramadan fasting.

Study Design: A cross-sectional study done at the outpatient clinic hospital at Universiti Sains Malaysia. Two hundred and forty three type 2 diabetes patients who attended the outpatient clinic were given a self-administered questionnaire. Two-way analysis of variance (2-way ANOVA) was used to test differences of the total mean score of knowledge, attitude, and practice between genders and between those who received health education and those who did not.

Result: Knowledge deficits were identified for symptoms of hypoglycemia, correct practice during fasting, and problems that may arise from fasting. A positive attitude was noted for self-control of the disease and the ability to fast. Medications were adjusted according to doctors' advice. The majority ate the predawn meal, but only $15.6 \%$ had supper, and $16.0 \%$ never took simple sugars on breaking fast.

Conclusion: Type 2 diabetes patients exhibited a good attitude toward Ramadan fasting despite deficits in knowledge and inappropriate practice.
\end{abstract}

Key words: Type 2 diabetes, Ramadan fasting, knowledge, attitude, practice.

Introduction

$\mathrm{R}^{\mathrm{s}}$ amadan fasting involves abstention from any oral intake, including medications, during the fasting hours. This voluntary practice causes changes in timing and frequency of eating meals and

Requests for reprints should be directed to

Nor Azwany Yaacob, MCommMed Lecturer, Department of Community Medicine

Health Campus, Universiti Sains Malaysia 16150 Kubang Kerian

Kelantan, Malaysia

Phone no.: 609 7663000; Fax no.: 6097653370

azwany@kb.usm.my taking medications. In 2001 in Malaysia, the average duration of daily fasting was 13.5 hours. Many diabetic Muslims have been observed to continue this practice in a clinical setting. Barber et al (1979) reported that half of the patients interviewed practice Ramadan fasting. ${ }^{1}$

Fasting diabetics should be educated about special problems that may occur during fasting and the necessary therapeutic adjustments. Fasting patients should be instructed not to skip meals, not to take medications irregularly, and not to gorge after the fast is broken. Dietary indiscretion during nonfasting hours contributes to the tendency to develop hyperglycemia and gain weight. ${ }^{2}$ Ramadan fasting benefits appear only in patients who maintain their appropriate diets. ${ }^{3}$ Patients on oral hypoglycemic medica- 
tions must adjust the dose properly. Patients treated with glibenclamide during Ramadan are recommended to switch the morning dose (together with any midday dose) of this drug to the dose taken at sunset. ${ }^{4}$ An educational program for diabetics during Ramadan should be directed toward 5

1. Diabetic home management

2. Preparing patients for Ramadan

3. Recognizing warning symptoms of dehydration, hypoglycemia, and other possible complications.

Currently, there is no specific program to educate diabetics on managing their disease during Ramadan. The diabetic health education program currently focuses on controlling the disease and disease complications. During clinical follow-up, the physician gives advice on how to safely fast during the month of Ramadan.

The purpose of this study was to assess the level of the patients' knowledge and their attitude toward Ramadan fasting, as well as how they adjust their medications, diet, and physical activity during the month. This information is thought to be useful to design an educational program for diabetics to follow during Ramadan.

\section{Materials and Methods}

Muslim diabetic patients who were fasting, were using an oral hypoglycemic agent(s), and attended either the Family Care Clinic (FCC) or the Diabetes Medical Clinic (DMC) during the month of Ramadan were studied. A sample group of 227 patients was estimated - using a single proportion formula based on a pilot study - to be of a sufficient size for the study. These patients were given self-administered questionnaires on sociodemographic and clinical characteristics. These characteristics were expressed by mean $(+\mathrm{SD})$ for numerical data and percentages for the categorical data. The chi square test was used to test the association of receiving health education with the type of clinic attended. The knowledge questions included questions on general health, diabetic complications, symptoms of hypoglycemia and hyperglycemia, and problems that may arise from fasting and safe Ramadan fasting. Questions on knowledge had three answer choices: "correct, "wrong," and "don't know". One point was given for each correct answer, and the knowledge score was computed by summing the score of all the knowledge statements. Questions on patients' attitudes included questions about the ability to control their disease state and their ability to carry out Ramadan fasting. Practice questions addressed the manner in which the patients practice their diets, drug intake, and physical activity during Ramadan. The answers to attitude and practice questions were given as five Likert scale options. For the attitude questions, the options were "strongly disagree," "disagree," "not sure," "agree," and "strongly agree". For the practice questions the options were "never," "seldom," "sometimes," "often." and "always".

The outcome measures were the knowledge mean scores of each subdomain, the proportion of patients who had a positive attitude and practiced Ramadan fasting correctly. The knowledge answer of "don't know" was combined with the wrong answer in order to more easily interpret the results. For the purpose of description of attitude, the scales designating agreement - "agree" and "strongly agree" - and the two scales designating disagreement - "strongly disagree", "disagree" - were combined into "agree" and "disagree" categories, respectively. Two-way analysis of variance (2-way ANOVA) was used to test differences of total mean score of knowledge, attitude, and practice between genders and between those who did and those who did not receive health education. The Universiti Sains Malaysia Ethical Committee approved the study according to the Helsinki Declaration.

\section{Results}

A total of 243 patients completed the questionnaire. There were $46.9 \%$ male and $53.1 \%$ female responders with mean age of 55.7 (9.31) years. The duration of years since diagnosis was 9.32 (6.70) (range 1-33 years). The majority of responders were Malays (98.8\%) and attended the DMC (60.3\%). Only $41.2 \%$ of responders claimed to have received health education on Ramadan fasting. Sixty-four percent of those were attending the DMC. However, there was no significant association between receiving Ramadan health education with type of clinic attended. More than half of the responders (59.9\%) have a family history of diabetes.

\section{Knowledge Score}

Figure 1 shows the mean total score of each knowledge subdomain. Each subdomain consists of three questions with maximum score of 3. A higher mean score was noted for questions on general 


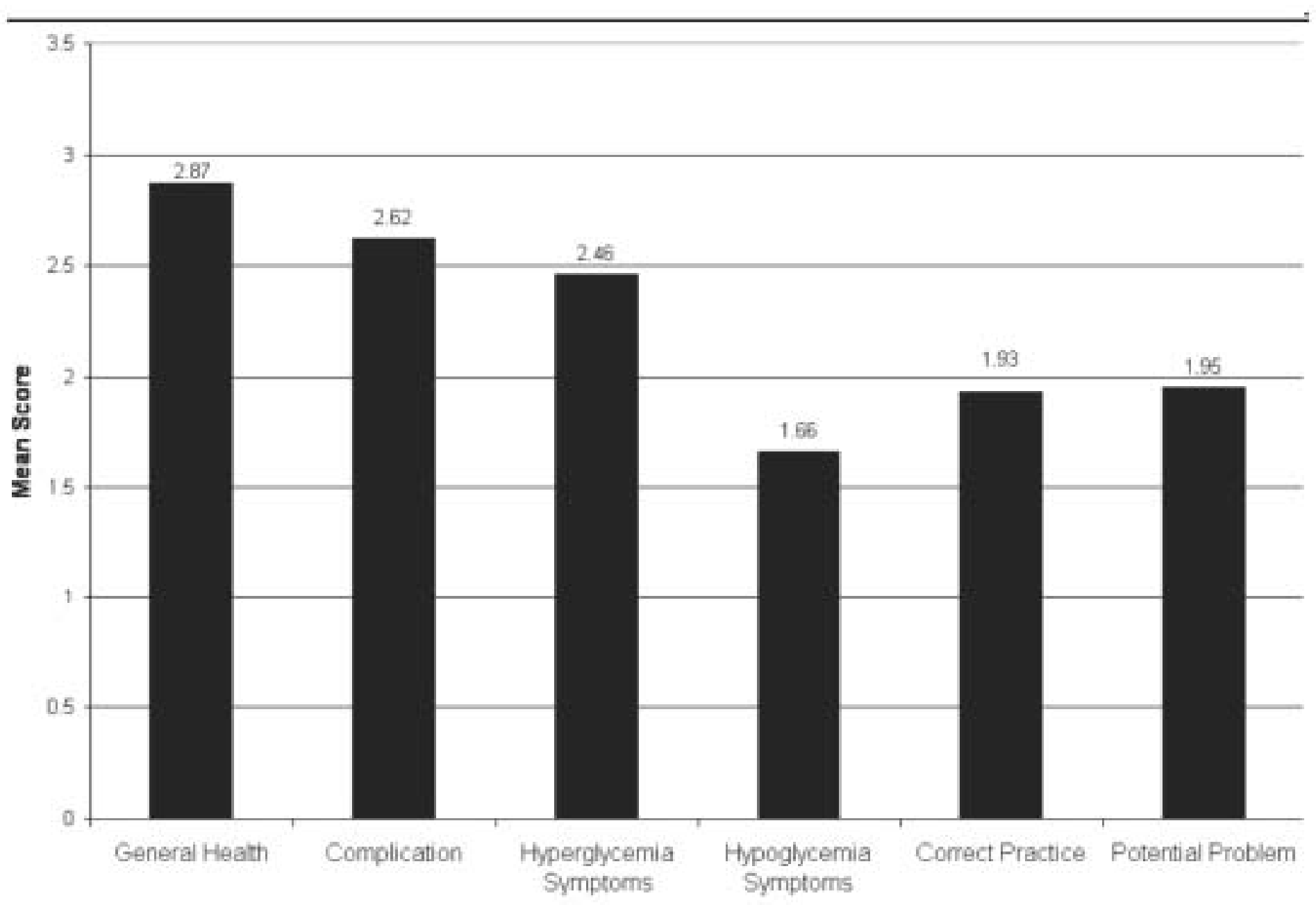

Figure 1. Mean score of knowledge domain.

health, diabetic complications, and symptoms of hyperglycemia. The mean score was relatively lower for questions regarding symptoms of hypoglycemia $(1.66+1.3)$, correct practice during fasting $(1.93+0.65)$, and problems that may arise from fasting $(1.95+0.7)$. There was no significant difference in the total mean score of knowledge between genders $(f=0.096$, $\mathrm{p}=0.76$ ) after controlling for the effect of receiving health education on Ramadan fasting. However, a significantly higher mean score was noted among those who had received health education compared to those who never received health education on Ramadan fasting ( $\mathrm{f}=6.711, \mathrm{p}=0.01$ ) (Table 1 ).

Many responders (81.9\%) said that having a heavy meal with rice or bread during the predawn meal (sahur) is necessary, and $18.9 \%$ responded that sweets (simple sugars) on breaking the fast is the key to compensate for the fasting glucose level. In terms of possible problems that may arise when fasting, only $36.2 \%$ knew the risk of dehydration. A majority of responders $(93.8 \%)$ knew that they should contin- ue their usual daily physical activity during Ramadan fasting, that fasting can help disease control (83.4\%), and that it does not increase the risk of getting infection (75.3\%).

\section{Attitude}

Table 2 shows the breakdown by percentage of responses to the attitude questions. A positive attitude was shown by high proportion of responders who agree on their responsibility for the control of disease (Questions 1 and 2). Similarly, a positive attitude was shown by their willingness and ability to carry out Ramadan fasting and their attitude towards physical activity (Questions 3, 5 and 6). There were still $11.5 \%$ of responders who were not sure that fasting could help disease control and another $4.5 \%$ who believed that fasting did not improve their disease control (Question 4). Males had a significantly better attitude with mean score of $24.7+2.1$, compared to females with a mean score of $23.9+1.8(f=8.43, p=0.004)$. However, there was no sig- 
Table 1. Effect of gender and receiving health education on total score of knowledge, attitude, and practice.

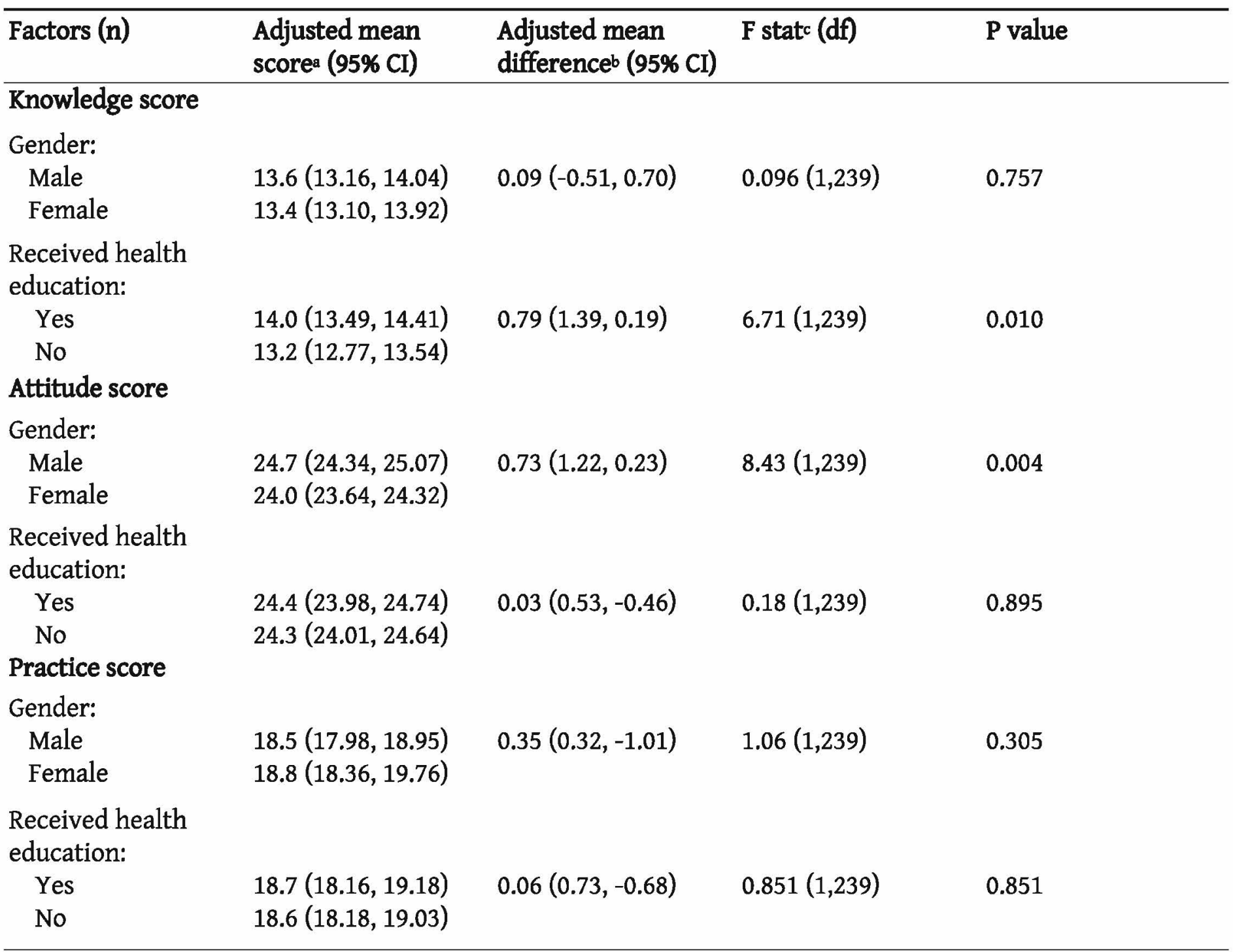

a Adjusted by the effect of gender or receiving health education

$b$ Bonferroni adjustment for $95 \%$ confidence interval for difference

c Two-way ANOVA

nificant difference in total mean score of attitude between those who had received Ramadan health education and those who did not (Table 1).

\section{Practice}

Table 3 shows the response to practice questions. A majority of responders discussed medication schedule adjustment with their doctor (83.1\%) and took the predawn meal (sahur) (98.8\%); however, there was still a high proportion of responders (58.4\%) who took sweet foods on breaking fast (sometimes, often, or always). There was a high proportion that did not have supper (66.2\%). There was no significant difference in the total mean score of practice between genders and those who received Ramadan health education and those who did not (Table 1).

\section{Discussion}

In a daily clinical setting, patients were given health education through direct discussion with the attending doctor or were sent to a dietician for dietary advice. Our study showed a high mean score of knowledge in terms of general health knowledge (ideal body weight, physical exercise, and dietary control) and diabetic complications i.e. renal, cardiac, and ophthalmic. The Diabetes Care Data Collection Project (DCDCP) on diabetes health educa- 
Table 2: Percentage of responses for attitude questions.

\begin{tabular}{llll}
\hline Question & Agree & Not sure & Disagree \\
\hline Q1. Should know blood glucose level & 98.4 & 0.4 & 1.2 \\
Q2. Able to control disease & 92.2 & 7.0 & 0.8 \\
Q3. Unable to fast because need to take medications & 2.1 & 1.2 & 96.7 \\
Q4. Fasting can help disease control & 84.0 & 11.5 & 4.5 \\
Q5. Ability to fast the whole month & 93.8 & 1.2 & 4.9 \\
Q6. Unable to do usual daily activity if fasting & 5.8 & 0.8 & 93.4
\end{tabular}

Table 3: Percentage of respondses for practice questions.

\begin{tabular}{llll}
\hline Question & Never and seldom & Sometimes & Often and always \\
\hline $\begin{array}{l}\text { Change medication sched- } \\
\text { ule based on doctor advice }\end{array}$ & 10.7 & 6.2 & 83.1 \\
$\begin{array}{l}\text { Reduced frequency of } \\
\text { medication }\end{array}$ & 53.5 & 4.5 & 42.0 \\
$\begin{array}{l}\text { Make sure take sahur } \\
\text { (predawn meal) }\end{array}$ & 0.4 & 0.8 & 98.8 \\
$\begin{array}{l}\text { Take sugary food on } \\
\text { breaking fast }\end{array}$ & 41.5 & 28.8 & 29.6 \\
$\begin{array}{l}\text { Take supper } \\
\text { akn }\end{array}$ & 66.2 & 18.1 & 15.6
\end{tabular}

tion profile in Malaysia reported $77 \%$ of patients in East Coast West Malaysia received health education on healthy eating, $12 \%$ on diabetic complications, and $13 \%$ on hypoglycemia. ${ }^{6}$ A high mean score on general health indicates the effect of the health education received. This study also showed a high mean score on diabetic complications, although the DCDCP report showed only a small percentage of patients had received health education. This may be due to the fact that self-experience and sharing the disease experience with other patients does increase their knowledge. Discussion with the attending doctor on lab studies, such as renal function tests, and referral to ophthalmologists also played a role in enhancing the knowledge of diabetic complications.

A relatively higher mean score of knowledge on hyperglycemia symptoms may be due to the fact that the patients had experienced it and presented with the symptoms on diagnosis. The patients also had experienced the symptoms when they were in a poorly controlled state. This is in line with the DCDCP report that showed $87.6 \%$ of East Coast West Malaysia diabetic patients were having fasting blood glucose (FBG) of more than $7.8 \mathrm{mmol} / \mathrm{L}$, and $92 \%$ were having HbA1C of more than $7.5 \% .^{6}$ Knowing how to recognize hyperglycemia symptoms can motivate patients to avoid compensatory eating and ingesting sweet, highly refined local foods that are available in abundance in this region during Ramadan. Avoiding compensatory eating and abstaining from high-calorie and highly refined foods are recommended in order to optimize control during Ramadan. ${ }^{7}$

A lower mean score of knowledge on hypoglycemia symptoms was noted despite a high mean score on hyperglycemia symptoms. Patients may have experienced symptoms but were not aware that these were warning symptoms of hypoglycemia. This finding was alarming and indicated the need for more 
health education so patients who want to carry out Ramadan fasting can recognize these symptoms to decide if to break the fast earlier or even abstain from fasting to prevent further complications and problems. ${ }^{8}$

Knowledge on dietary practice during Ramadan was also relatively poor. Many responders (81.9\%) answered that they must take a heavy meal with rice or bread during the predawn meal (sahur). The other responders, $18.9 \%$, believed they must take sweet, highly refined sugary foods on breaking fast to compensate for the "fasting" glucose level. These findings were noted despite a good knowledge on the importance of dietary control, which suggests that current knowledge was not adequate for the patients to practice Ramadan fasting appropriately.

There were still $16.0 \%$ who did not know that Ramadan fasting helps to control diabetes. Previous studies have shown that fasting Ramadan is associated with improvement of diabetic control rather than worsening it.,8,9 This should be stressed so patients will be more motivated to control their diet and drug intake during Ramadan in order to maintain good diabetic control.

Patients with poorly controlled diabetes may lose excessive water because of high blood glucose levels. When the kidneys cannot conserve water, the patient will suffer from dehydration. This is worsened if the patient continues to fast. ${ }^{7}$ Knowledge about the possible risk of dehydration is important so that the patients will drink an adequate amount of water during the nonfasting period along with maintaining good diabetic control. Only a small proportion of patients $(36.2 \%)$ was aware of this risk. Doctors should emphasize the importance of adequate water intake, especially to elderly patients and to those who are actively working outdoors during the day.

There was a quite high proportion of responders (24.7\%) who thought that fasting could increase the risk of infections. Patients should be told that infection risk is associated with inadequate glucose control ${ }^{10}$ and not directly from fasting. This point should be emphasized together with the importance of maintaining good diabetic control during the month. A majority of patients know they can continue their daily physical activity (93.8\%). This may be related to the fact that most responders were feeling better when practicing Ramadan fasting. It is also impor- tant to recommend that diabetic patients continue their usual physical activity, especially during the nonfasting periods. ${ }^{7}$

A majority of responders had a positive attitude toward their own responsibility to control and monitor their disease and their willingness and ability to carry out Ramadan fasting. This correlates well with the fact that many diabetic patients practice Ramadan fasting. The positive attitude on disease control and monitoring can be a good start to encourage more patients to do home blood glucose monitoring. The Diabetes Care Data Collection Project reported that only $0.4 \%$ of patients in East Coast West Malaysia do home blood glucose monitoring. ${ }^{6}$ Self-monitoring of blood glucose levels will safeguard against serious fluctuations of the blood glucose level during the day, especially during the first week of Ramadan.

Proper adjustment in the dosage of hypoglycemic agents will lower the risk of hypoglycemia and hyperglycemia in fasting diabetic patients. Although many responders discussed this with their doctors, there were some responders who adjusted the schedule without consulting their caregiver. Those who reduced their medications did so either on the advice of their doctor for convenience of taking medication during the nonfasting period or when they developed hypoglycemic symptoms. Those who did not reduce their drug frequency schedule were mainly on twice-daily or once-daily doses of medication. Even though responders adjusted their medication regime after discussing it with their doctor, patients should be encouraged to do home glucose monitoring to ensure good diabetic control.

Taking the predawn meal and a late night meal immediately before sleep has been advised to help prevent possible episodes of hypoglycemia. Many of the responders were aware of the need for taking the predawn meal at the same time they were taking their morning dose of medication. However, few responders ate late at night before sleeping, mainly because they were still feeling full and because it was not their usual habit. Thus, this should be emphasized to those who take their second dose of drug before going to bed.

More than half of the study subjects still took highly refined sugars on breaking fast. This finding is similar to another study at Hospital Universiti Sains 
Malaysia (HUSM), which reported a significant increase in the intake of refined sugars contained in local delicacies by diabetic patients during Ramadan, ${ }^{3}$ and a study in Riyadh, Saudi Arabia, which reported among the study participants a tendency to overeating during nonfasting hours. ${ }^{9}$

In conclusion, our study identified knowledge deficits, misconceptions, and inappropriate practice among type 2 diabetic patients who practice Ramadan fasting. Therefore, a specific health education program on how to carry out a safe and healthful Ramadan fasting is essential in addition to the current health education program for diabetic patients. Ramadan health education program must include dietary advice; drug regime adjustment; recognizing symptoms of dehydration, hypoglycemia, and hyperglycemia; and the importance of breaking fast as soon as any complication occurs. They also should be informed on how to seek help if a problem occurs.

\section{Acknowledgements}

We would like to thank the Intensified Research for Priorities Area short-term research grant for Universiti Sains Malaysia for the support of this study.

\section{References}

1. Barber SG, Fairweather S, Wright AD, et al.
Muslims, Ramadan, and diabetes mellitus. Br Med J. 1979;2(6181):46-7.

2. Rasheed $\mathrm{AH}$. The fast of Ramadan. Br Med J. 1992;304(6826):521-2.

3. Mafauzy M, Mohammed WB, Anum MY, et al. A study of the fasting diabetic patients during the month of Ramadan. Med J Malaysia. 1990;45(1):14-7.

4. Belkhadir J, el Ghomari $\mathrm{H}, \mathrm{Klocker} \mathrm{N}$, et al. Muslims with non-insulin dependent diabetes fasting during Ramadan: treatment with glibenclamide. Br Med J. 1993;307(6899):292-5.

5. Shahid A. 2001. Diabetes mellitus and Ramadan fasting. Available at http://www.islam-usa.com.

6. Malaysian Endocrine and Metabolic Society, Ministry of Health, Malaysia, Malaysian Diabetes Association. National Action Plans and Strategies: Diabetes Care Data Collection Project. Proceedings of National Diabetes Care Seminar, 1998.

7. Malaysian Diabetes Association. Diet and diabetes. Pelanduk Publication: Petaling Jaya. 1993.

8. Azizi F and Siahkolah B. Ramadan fasting and diabetes mellitus. Int J Ramadan Fasting Res, 1998;2:817.

9. Laajam MA. Ramadan fasting and non-insulindependent diabetes: effect on metabolic control. East Afr Med J. 1990;67(10):732-6.

10. Rayfield EJ, Ault MJ, Keush GT: Infection and diabetes: the case for glucose control. Am J Med. 1982;72(3):439-50.

\title{
The Islamic Medical Association of North America (IMANA) and the Federation of Islamic Medical Associations (FIMA) Need Academic Faculty
}

\author{
FIMA has been approved by the Economic and Social Council of the United Nations \\ (ECOSOC) for "Special Consultative Status."
}

We will be embarking on a new mission where our member organizations, including IMANA, will play a major role.

Kindly send your CV to:

\author{
Prof. Muhammed A.A.Khan, MD \\ Professor Emeritus \\ Executive Director-FIMA \\ email: maakhan_60521@yahoo.com \\ cc also to hq@imana.org
}

